Review Article

\title{
MESOIONIC SYDNONE: A REVIEW IN THEIR CHEMICAL AND BIOLOGICAL PROPERTIES
}

\author{
ABDUALRAHMAN MOHAMMED ABDUALKADER ${ }^{1}{ }^{*}$, MUHAMMAD TAHER ${ }^{2}$, NIK IDRIS NIK YUSOFF1
}

1Pharmaceutical Chemistry Department, ${ }^{2}$ Pharmaceutical Technology Department, Faculty of Pharmacy, International Islamic University

Malaysia, Kuantan, Pahang, Malaysia

Email: armak1983@gmail.com

Received: 08 Mar 2017 Revised and Accepted: 19 Jun 2017

\section{ABSTRACT}

Various literature sources have documented sydnones as important molecules with exclusive chemical properties and a wide spectrum of bioactivities. Sydnone can be defined as a five-membered pseudo-aromatic heterocyclic molecule. Classically, 1,2,3-oxadiazole forms the main skeleton of sydnone. The molecule has delocalized balanced positive and negative charges. The five annular atoms share the positive charge and the enolate-like exocyclic oxygen atom bears the negative charge. The hydrogen atom at the position C4 was proved to have acidic and nucleophilic functionalities making the sydnone ring reactive towards electrophilic reagents. These unique chemical features enable sydnones to interact with biomolecules resulting in important therapeutic effects like anticancer, antidiabetic, antimicrobial, antioxidant and anti-inflammatory. Consequently, we aim from the current article to review the available chemical and pharmacological information on sydnone and its derivatives.

Keywords: Sydnone, Mesoionic, Heterocycles, Anticancer, Antimicrobial, Anti-inflammatory

(C) 2017 The Authors. Published by Innovare Academic Sciences Pvt Ltd. This is an open access article under the CC BY license (http://creativecommons.org/licenses/by/4.0/) DOI: http://dx.doi.org/10.22159/ijpps.2017v9i8.18774

\section{INTRODUCTION}

Sydnones are the most studied compounds amongst the mesoionic family due to their interesting structures, chemical properties, synthetic utility and biological activities. Many reports stated that one covalent structure is not sufficient to represent the sydnone molecule satisfactorily $[1,2]$. However, 1,2,3-oxadiazolium bearing a carbonyl function has recently been the major representative of sydnones because FTIR spectroscopy showed a carbonyl stretch frequency attached to $\mathrm{C} 5$ of the ring like in 4-acetyl-3-tolylsydnone which exhibited a strong band at $1783 \mathrm{~cm}^{-1}$ [3]. X-ray analysis revealed a bond length of $1.196 \AA$ which corresponds to an exocyclic $\mathrm{C}=0$ double bond [4]. Classically, the sydnone ring can be prepared from the cyclization of $\mathrm{N}$-nitroso amino acids with acetic anhydride [5]. Later, many attempts were employed to improve the yield of cyclization by using a stronger dehydrating agent such as trifluoroacetic acid anhydride or thionyl chloride [6]. Since their first preparation, sydnones attracted the attention of medicinal chemists and pharmacologists to investigate their biological applications. Their distinguished chemical structure enables them to bind and deactivate a variety of biomolecules like DNA and enzymes. A vast range of therapeutic properties has been demonstrated including antimicrobial, anti-inflammatory, anti-cancer, antioxidant and antidiabetic [7]. The present review demonstrates the important chemical and biological data on sydnones starting from their early discovery in 1935 until today.

\section{Chemistry of sydnone}

\section{Definition of sydnone}

The word sydnone was originated from the phrase "University of Sydney" where this class of compounds was first prepared by Earl and Mackney in 1935. They suggested the formation of fused threeand four-membered ring product (I) from the action of acetic anhydride on $N$-nitrosophenylglycine [5] which was later considered wrong by other chemists.

Firstly, a fused ring system is unlikely to be formed by a simple intramolecular rearrangement and would be a highly strained unstable structure due to the existence of a $\beta$-propiolactone group. Therefore, Baker and his collaborator omitted the bridge bond and recommended a partially aromatic five-membered ring (II and III) which was a hybrid of many zwitterionic forms [8]. Secondly, acid hydrolysis decomposes sydnone into hydrazine, carboxylic acids and carbon dioxide while hot aqueous sodium hydroxide can revert the sydnone into the starting $N$-nitroso compound. These two facts indicate that the bicyclic system proposed by Earl is improbable [9]. Thirdly, other researchers proved that acetic anhydride can convert the dextro-rotary $N$-nitroso- $N$-phenylalanine into the optically inactive $N$-phenyl- $C$-methylsydnone (IV). The loss of optical activity implies either racemization or a change in the hybridization of $\mathrm{C} 4$ from a chiral $\mathrm{sp}^{3}$ state into an achiral $\mathrm{sp}^{2}$. The oxygen atom attached to $\mathrm{C} 5$ was proved to be in an enolate form due to the rapid formation of a monobromo derivative (V) in glacial acetic acid and bromine [10].

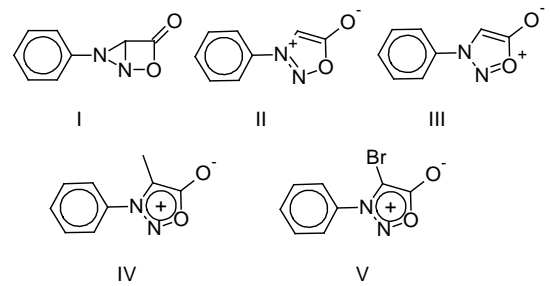

To put a limit to the previous debate, Baker and Ollis extensively reviewed all proposed structures of sydnone and suggested more clear description as follows:

1. A single covalent structure from the preceding suggestions does not fairly describe sydnone. In other words, the sydnone molecule should be considered as a hybrid of bipolar and tetrapolar forms (VI$\mathrm{XI}$ ) whose contribution to the hybrid ring is not equal. Consequently, sydnones are described as mesoionic compounds.

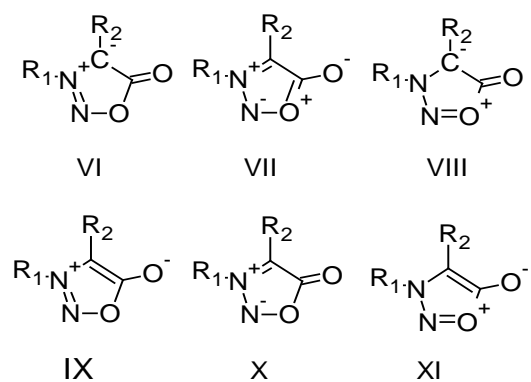

2. Compared to cyclopentadienyl anion (XIIa, XIIb), tropylium cation (XIIIa, XIIIb), furan, pyrrole and pyridine, the sydnone ring 
has all requirements to develop aromatic properties. The exocyclic oxygen atom provides an electron to the ring to complete the sextet of $\pi$ electrons. Moderately satisfied, they came up with the formula $\mathrm{XIV}$ to represent the sydnone structure.

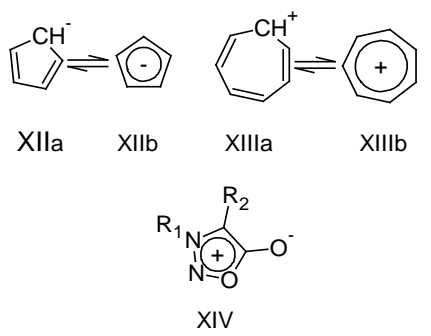

After all, they defined sydnone as a mesoionic compound consisting of a five-membered aromatic heterocyclic ring mainly 1,2,3oxadiazole or a six-membered ring in some cases. The molecule is neutral and has a positive charge in common between the annular atoms balanced by a negative charge borne on an exocyclic atom(s). Even though there is no single polar or covalent structure for sydnone, the structure XIV is being used as a representative in the majority of literature up to date $[1,2]$.

\section{Synthesis of sydnone}

Primarily, sydnone was prepared by Earl and his colleague by the cyclodehydration effect of acetic anhydride on the $N$-nitroso derivatives of amino acids. They reported that the dissolution of $\mathrm{N}$ nitroso- $N$-arylglycine in excess acetic anhydride at room temperature resulted, after $24 \mathrm{~h}$, in a nitroso-free, crystalline and stable heterocyclic product which was later referred to as sydnone. The preparation of the $N$-nitroso intermediate was accomplished by the conventional nitrosation of the amino group of $N$-phenylglycine by the nitrous acid generated from the reaction of sodium nitrite and hydrochloric acid [5]. The $N$-nitrosation of $N$-phenylglycine in neutral conditions was described later by Applegate and Turnbull using isoamyl nitrite (IAN) in dimethoxyethane (DME) at room temperature (Scheme 1). They claimed that IAN was successfully used to prepare the $N$-nitroso derivative of $N$-(2-acetylphenyl) glycine with high yield compared to the acid-based method which led to the formation of $C$-nitroso glycine [11].

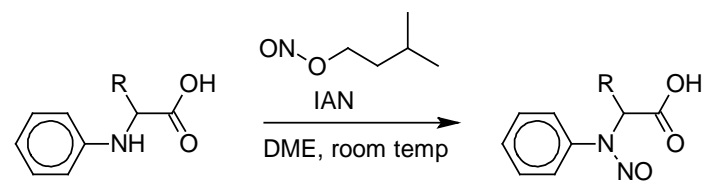

Scheme 1: Preparation of $N$-nitroso analogues in neutral conditions

Later, Baker et al. deduced the mechanism of cyclization of the $\mathrm{N}$ nitroso starting material by losing a water molecule which involves four steps as presented in Scheme2. Firstly, a mixed anhydride intermediate XV will be formed from the effect of acetic anhydride on the free nitroso acid whose carbonyl group will evolve strong cationic properties. Of interest, it was found that using a potassium salt of the $N$-nitroso- $N$-phenylglycine will drastically slow down the development of the intermediate. Secondly, a nucleophilic attack of the nitroso oxygen on the acid carbonyl group will lead to ring closure (XVI). Thirdly, an acetate group is lost, and a double bond between the two nitrogen atoms is formed (XVII). Lastly, loss of proton and formation of enolic oxygen will produce the final sydnone product XVIII [6].

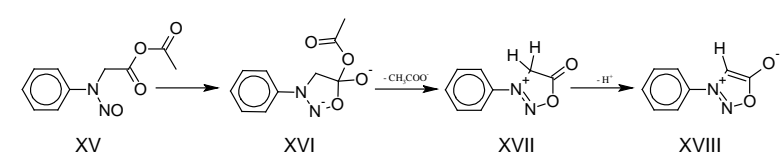

Scheme 2: Mechanism of ring closure and sydnone formation
However, Eade and Earl found that the preparation of some sydnone analogues such as nitro-containing sydnone took a long time up to 7$30 \mathrm{~d}$ at room temperature with low to moderate yields. They claimed that heating accelerated the formation of sydnone ring, even though it reduced the yield due to rapid hydrolysis of the product by the hot acidic reaction medium [9]. Therefore, Baker and his colleagues reported for the first time an instant and complete separation of $\mathrm{N}$ arylsydnone in $90 \%$ yield when they used trifluoroacetic anhydride (TFAA) as a dehydrating agent [6]. The later synthetic route had been successfully utilized to prepare some complicated sydnones such as $N, N$-polyaliphatic bis-sydnone at a yield of $70-80 \%$ [12]. Moreover, heat-labile sydnone such as 3-(2methoxycarbonylphenyl) sydnone was prepared in a considerable

yield of $75 \%$ within one hour using TFAA in dichloromethane at $5{ }^{\circ}$ $\mathrm{C}$ [13]. Many alternative reagents were also employed to prepare the sydnone system. In 1950, Baker et al. used thionyl chloride. They reported that the conversion of $N$-nitroso amino acids into sydnone took place within a few minutes using thionyl chloride in dry ether at room temperature giving a low yield of $28 \%$. On the other hand, using thionyl chloride in a mixture of cold dioxane and pyridine resulted in an improved yield (75\%) within 25 min [6].

Some special structures of sydnones were reported as unexpected products of the cyclodehydration of the $N$-nitroso derivatives of $\alpha \alpha^{\prime}$ iminodicarboxylic acids. For example, 4, 4'-methylene bis [3-(2cyanoethyl) sydnone] XIX was obtained from the effect of acetic anhydride on the diastereoisomeric mixture of $\alpha \alpha^{\prime}-d i-(N-2$-cyanoethyl$N$-nitrosoamino) glutaric acid XX. On the contrary, the individual $\alpha$ and $\beta$ forms gave mainly the cyclic anhydride; $N$-2-cyanoethyl $N$-nitroso-Lglutamic anhydride XXI [14]. Likewise, the hydrolysis of the latter cyclic anhydride in water at room temperature led to an intramolecular rearrangement giving 3-(2-cyanoethyl)-4-(2carboxyethyl) sydnone XXII with $35 \%$ yield [15].

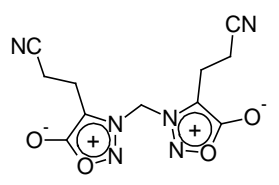

XIX<smiles>N#CCCN(C1CC(N(CCC#N)[N+](=O)[O-])C(=O)OC1=O)[N+](=O)[O-]</smiles>

XXI<smiles>[X][N+]([O-])(CC(C(=O)O)N(CCC#N)[N+](=O)[O-])N([O-])CCC#N</smiles>

$X X$<smiles></smiles>

XXII
Recently, $\quad N, N, N^{\prime}, N^{\prime}$-tetrabromobenzene-1,3-disulfonamide XXIII (TBBDS) and 1,3-dibromo-5,5-dimethylhydantoin XXIV (DBH) were employed as catalysts for the one-pot conversion of $N$-arylglycines into the corresponding sydnone in a neutral medium. Stirring of $\mathrm{N}$ arylglycine, TBBDS, sodium nitrite, and acetic anhydride in dichloromethane (DCM) at $5{ }^{\circ} \mathrm{C}$ was sufficient to bring about the formation of the sydnone ring in a very satisfactory yield during 5-8 $\mathrm{h}$ [16]. Similarly, DBH efficiently promoted the cyclization within 10$16 \mathrm{~h}$ [17]. Worth mentioning, sydnones containing nitrophenyl groups were prepared in a yield of $80-88 \%$ using the previous reagents compared to $5-30 \%$ by the classic Earl's method $[9,16,17]$.<smiles>O=S(=O)(Br)c1cccc(S(=O)(=O)N(Br)Br)c1</smiles>

XXIII<smiles>CC1(C)C(=O)N(Br)C(=O)N1Br</smiles>

XXIV

\section{Physicochemical properties of sydnone}

\section{The electronic structure of sydnone ring}

Using a new method of molecular orbital calculation named as $\omega_{-}$ technique modified from Hückel framework, Kier and Roche calculated 
reasonable values of charge densities and bond orders for 3methylsydone and 3-phenylsydone. The calculated bond order value of C5-06 bond in structure XXV and XXVI [18] along with the X-ray structure of 3-(p-bromophenyl) sydnone [19], 3-(p-ethoxyphenyl) sydnone and 3-( $p$-tolyl)sydnone [20] pointed out a carbonyl-like double bond characters of the $\mathrm{C} 5-\mathrm{O} 6 \mathrm{~b}$ bond of the sydnone ring. Moreover, it can be noticed that the exocyclic oxygen 06 is highly negative charged ($0.53)$ even stronger than the carbonyl oxygen of butyrolactone $(-0.38)$ indicating a very polarizable carbonyl group [18].
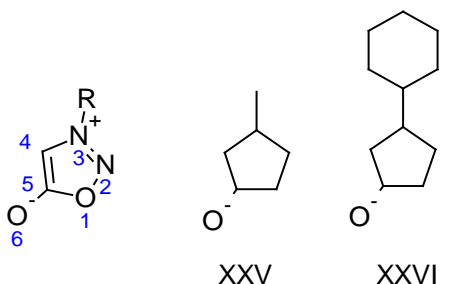

The delocalized positive charge of the ring which has been previously proved to be unevenly distributed is mainly borne on the number three nitrogen especially when an electron withdrawing group is attached to the nitrogen such as a phenyl ring. This suggests an iminium-type nature of this nitrogen and therefore has an electron withdrawing impact which will deactivate the attached aryl substituents towards all electrophilic reagents [18]. Even though, the calculated bond order of the $\mathrm{N}-0$ was $1.22 \AA$ which was close to $1.14 \AA$ of the double bond $\mathrm{N}=\mathrm{O}$, the $\mathrm{X}$-ray confirmed its single bond nature $[19,20]$. Interestingly, the high electron density of C4 in structures XXV and XXVI was later supported by the finding of Greco and O'Reilly who reported a strong acidity of 3-phenylsydnone with $\mathrm{p} K_{\mathrm{a}}$ value of 18-20. Consequently, sydnone is an electron donating reagent and the electrophilic substitution at this position is possible [21].

However, more recent studies by Fan and his lab-mates gave a better insight into bond length and nature, atomic charges and electron density distribution for thirteen different sydnone compounds. Ring aromaticity is gained from the unequally delocalized $\pi$-electrons with higher density on both N2-N3 and N3-C4. The calculated bond length of C5-06 (1.80-1.85 $\AA$ ) and the experimental value (1.19-1.22 A) illustrated undoubtedly its double bond nature. On the other hand, C5-01, N2-01 and C4-C5 were mainly single bonds. The other bonds of the ring, N2-N3 and N3-C4 were found partially double bonds. The net atomic charges were summarized as follows: neutral $(\mathrm{N} 2, \mathrm{~N} 3)$, positive $(\mathrm{C} 5)$ and negative $(01, \mathrm{C} 4,06)$ with the highest negative potential $(-84.6 \mathrm{kcal} / \mathrm{mol})$ was deposited at 06. Conjugation between sydnone ring and the aromatic system substituted at N3 was not essential for the stability of sydnones because the resonance between the two systems was minor [22].

\section{Sydnones spectral studies}

\section{Ultraviolet (UV) spectroscopy}

The properties of the ultraviolet spectra of sydnones were well reviewed by Stewart (23) and Kier and Roche (24). Briefly, absorption maxima in the range $290-340 \mathrm{~nm}$ was considered as a proof of the presence of the aromatic ring of sydnone. Alkyl sydnone absorbs at the lower wavelength $(<300 \mathrm{~nm})$. For example, 3methylsydnone, 3-n-butyl sydnone and 3-cyclohexylsydnone showed their UV absorption maxima at 290, 289.5 and $292 \mathrm{~nm}$, respectively. A bathochromic shift was observed for 3-arylsydnone due to conjugation as in 3-phenylsydnone and 3-(1-naphthyl) sydnone which absorb at 310 and 315, respectively. Many factors can remarkably affect the UV spectra of sydnones:

1. Conjugation: An aromatic system substituted at $\mathrm{C} 4$ of the sydnone ring has a stronger bathochromic effect such as 3-methyl-4phenylsydnone whose UV maxima was at $317 \mathrm{~nm}$. Similarly, 4acetylated sydnone absorbs at a longer wavelength such as 4-acetyl3-phenylsydnone and 3-phenylsydone absorb at 324 and $310 \mathrm{~nm}$.

2. Steric factors can retard the conjugation due to the disturbance of the planarity of the molecule. The UV maxima of $3-(2,6-$ methylphenyl) sydnone was found to be at $255 \mathrm{~nm}$ even shorter than that of 3-alkyl sydnone which lacks conjugation.
3. Electrostatic interaction in bis-sydnone system makes the coplanarity system more rigid and therefore the UV absorption wavelength is unusually high like in XXVII, XXVIII, XXIX and XXXI whose maximum absorptions were at 292,350, 292 and 303, respectively.
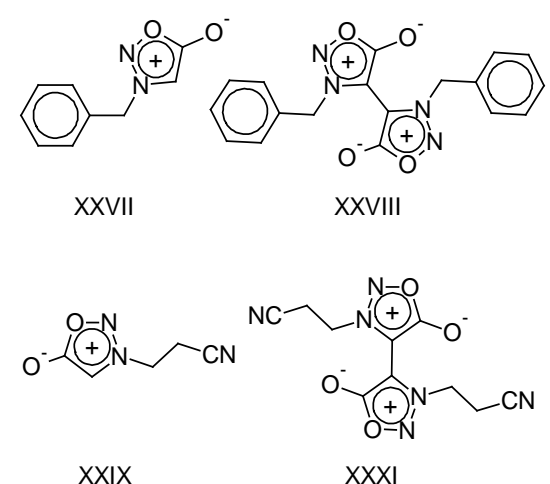

\section{Infrared (IR) spectroscopy}

A survey of the literature since their early preparation until today revealed two characteristic IR bands for sydnones. The stretch of sydnone carbonyl (C5-0) ranges from 1740 to $1770 \mathrm{~cm}^{-1}$ while the absorption band of carbon-hydrogen (C4-H) was more than 3000 $\mathrm{cm}^{-1}$. However, electrophilic substitution at C4 led to the loss of the carbon-hydrogen band and an increase in the wavenumber of the carbonyl up to $1780-1830 \mathrm{~cm}^{-1}$. For example, acetylation of 3-(4chlorophenyl) sydnone resulted in upshifting the CO band from $1750 \mathrm{~cm}^{-125}$ to $1786 \mathrm{~cm}^{-13}$.

\section{Nuclear magnetic resonance (NMR) spectroscopy}

Lawson et al. observed an unusually high field resonance of the sydnone ring proton in a series of 3-alkylsydnone and 3-arylsydnone when compared to the normal olefinic hydrogen. They confined this deshielding phenomenon to the electron-withdrawing effect of the adjacent nitrogen and oxygen atoms along with the anisotropy effect of the nearly coplanar phenyl ring. Likewise, the positive charge and the aromatic features of the sydnone ring resulted in deshielding the phenyl ring protons, especially the hydrogen atoms on the carbon $\alpha$ to the sydnone ring [26]. Another characteristic component of the sydnone NMR spectra is the peak of the carbon C5 which appears in the lowest field of ${ }^{13} \mathrm{C}$ NMR spectrum, i.e. the carbonyl region. The chemical shift of sydnone $\mathrm{CO}$ peak changes within a narrow range from 160-170 ppm regardless of the nature of the substituent at C4. As examples, compounds XXXII, XXXIII, XXXIV have their CO peaks at $169.19 \mathrm{ppm}$ [27], $165.80 \mathrm{ppm}$ [3] and $163.20 \mathrm{ppm}$ [28].
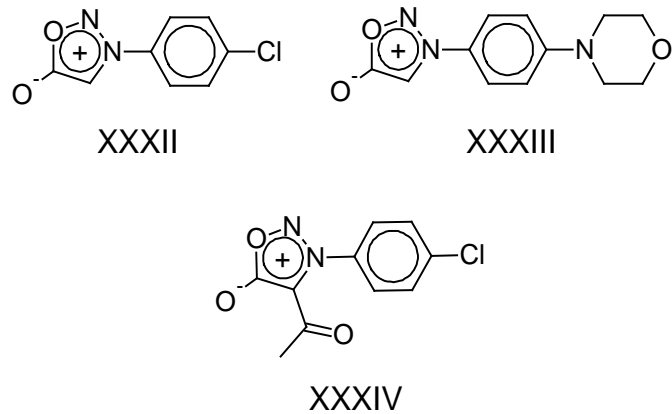

\section{Chemical properties of carbon $\mathrm{C} 4$ of the sydnone ring}

\section{Acylation and formylation of sydnone ring}

Greco and his co-workers reported unsuccessful attempts to acylate the sydnone ring in the conventional Friedel-Crafts reaction using many catalysts such as aluminum chloride, stannic chloride, and phosphoric acid. However, they successfully prepared a variety of 4acylsydnone derivatives when phosphorous pentoxide (3 equiv) was 
refluxed with one molar equivalent of carboxylic acid and sydnone [29]. Later, other chemists speculated that the failure of FriedelCrafts acylation was due to the coordination between Lewis acid and the exocyclic oxygen of the sydnone which eventually yielded a sydnone-containing fused ring compounds rather than the desired acylated product as shown in Scheme 3 [30]. More studies on the acylation of sydnone were later conducted employing a wide range of catalysts with a moderate to excellent yield as summarized in table 1.<smiles>[R]C(=O)CCn1cc([O-])nn1</smiles>

Scheme 3: Formation of a fused-ring sydnone via direct acylation of the sydnone ring

Table 1: Acylation of 3-arylsydnone

\begin{tabular}{|c|c|c|c|}
\hline Catalyst & Experimental & Yield (\%) & Reference \\
\hline $\begin{array}{l}\text { Bis (triphenylphosphine)palladium(II) dichloride } \\
\mathrm{PdCl}_{2}\left(\mathrm{PPh}_{3}\right)_{2}\end{array}$ & $\begin{array}{l}\text { Arylsydnone-4-yl copper, acyl chloride and the } \\
\text { catalyst for } 2 \mathrm{~h} \text { at room temperature }\end{array}$ & $50-95$ & {$[31]$} \\
\hline Montmorillonite K-10 & Reflux overnight in acetic anhydride & $25-86$ & {$[32]$} \\
\hline 1,3-Dibromo-5,5-dimethylhydantoin (DBH) & Reflux $7 \mathrm{~h}$ in acetic anhydride & $80-90$ & [3] \\
\hline N-Bromosuccinimide (NBS) & Reflux $4 \mathrm{~h}$ in acetic acid at $110{ }^{\circ} \mathrm{C}$ & $90-95$ & [33] \\
\hline $\begin{array}{l}\text { Bismuth(III) trifluoromethanesulfonate } \mathrm{Bi}(\mathrm{OTf})_{3} \text { and lithium } \\
\text { perchlorate } \mathrm{LiOCl}_{4}\end{array}$ & $\begin{array}{l}\text { Heating an alkyl anhydride with the catalysts in } \\
\text { acetonitrile at } 95^{\circ} \mathrm{C} \text { for } 2-16 \mathrm{~h}\end{array}$ & $48-93$ & {$[34]$} \\
\hline Indium(III) trifluoromethanesulfonate $\operatorname{In}(\mathrm{OTf})_{3}$ & $\begin{array}{l}\text { Overnight heating at } 80^{\circ} \mathrm{C} \text { in the presence of acetic } \\
\text { anhydride in acetonitrile }\end{array}$ & $40-80$ & {$[35]$} \\
\hline $\begin{array}{l}\text { Bismuth(III) trifluoromethanesulfonate } \mathrm{Bi}(\mathrm{OTf})_{3} \text { and lithium } \\
\text { perchlorate } \mathrm{LiOCl}_{4}\end{array}$ & Microwave irradiation for $15-45$ min & $11-95$ & {$[36]$} \\
\hline
\end{tabular}

On the other hand, formylation of sydnone ring at $\mathrm{C} 4$ was conducted using the classic Vilsmeier reaction with a yield up to $50 \%$. Noteworthy, formylation occurred only at the sydnone ring, and no formyl group was found at the substituted phenyl ring [37]. Later, Yeh and his colleagues used the formylated sydnone to prepare 4carbamoyl or 4-cyano sydnone from the reaction of 4-formyl-3arylsydnone with concentrated or dilute sulfuric acid, respectively, in the existence of sodium azide [38].

\section{Halogenation of sydnone ring}

Many methods were developed for the introduction of halogens into carbon C4 of the sydnone ring. Chloro, iodo and bromo substituted sydnones were prepared successfully with a satisfactory yield as summarized in Scheme 4 [39-42]. To date, fluoro-containing derivatives at C4 have not been reported. However, Foster and his co-workers reported 4-trifluoromethyl-3-arylsydnone from 3,3,3-trifluoro-2-( $N$ nitroso arylamino)propanoic acid with $75-85 \%$ yield [28].

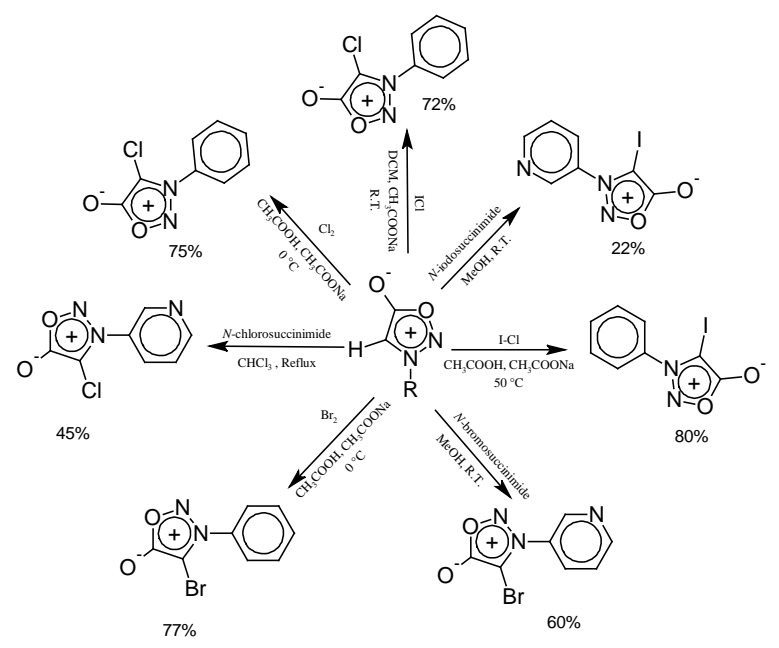

Scheme 4: Halogenation of sydnone at carbon C4

\section{Sydnone lithiation and its application}

The preparation of 3-aryl-4-lithiosydnone is straightforward and can be achieved by $n$-butyllithium in tetrahydrofuran (THF) within one hour. Fuchigami and others reported that the 4-sydnonyl anion generated from 4-lithiosydnonein THF could react easily and selectively with various chemicals to introduce heteroatom groups at the C4 position as a sole product with an excellent yield. They found that 4-sydnonyl anion had less nucleophilicity features than the ordinary aryl anion and therefore the reaction with phosphorous acid esters, tin (II) chloride and antimony trichloride was not successful as presented in Scheme $5[43,44]$.

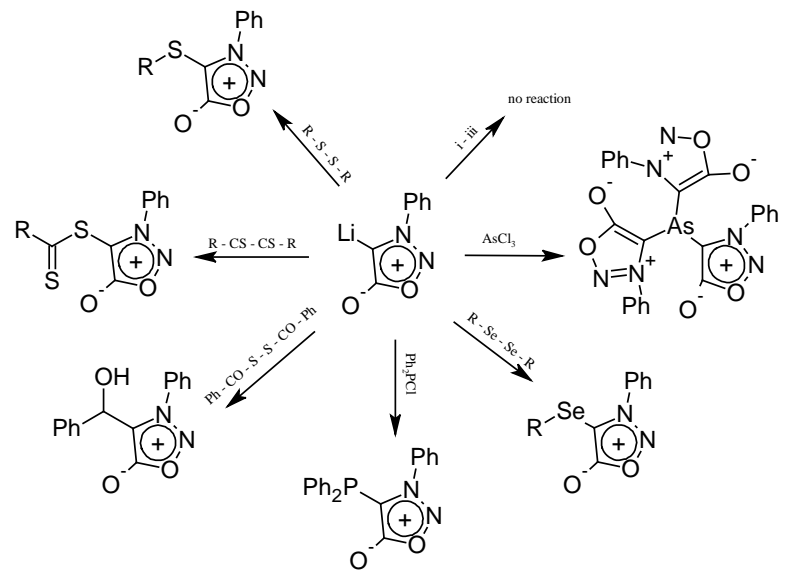

Scheme 5: The introduction of heteroatom groups to 3arylsydnone via 4-lithiosydnone as an intermediate. All reactions were performed at $-50{ }^{\circ} \mathrm{C}$. i) tin (II) chloride $\mathrm{SnCl}_{2}$, ii) antimony trichloride $\mathrm{SbCl}_{3}$, iii) trimethyltin chloride $\left(\mathrm{CH}_{3}\right)_{3} \mathrm{SnCl}$

Later, dilithium species (4-lithio-o-lithio arylsydnone) were reported for the first time by reacting 3-arylsydnone with 2.2 equivalents of $n$-butyllithium and tetramethylethylenediamine (TEMEDA) in THF at-78 ${ }^{\circ} \mathrm{C}$. The di-lithiated product was subsequently subjected to a variety of electrophiles to produce the disubstituted sydnone such as di-formyl, di-trimethylsilyl, di-halo, di-alkyl, di-thioether $[45,46]$ or fused sydnone rings such as 4hydroxy-4-substituted sydno(3,4-a)(4H)indoles [47]. Also, the acylation of 3-arylsydnone at the ortho-aryl position was accessed by adding Weinreb amides directly to the reaction pot of dilithium analogues as shown in Scheme 6 [48]. 


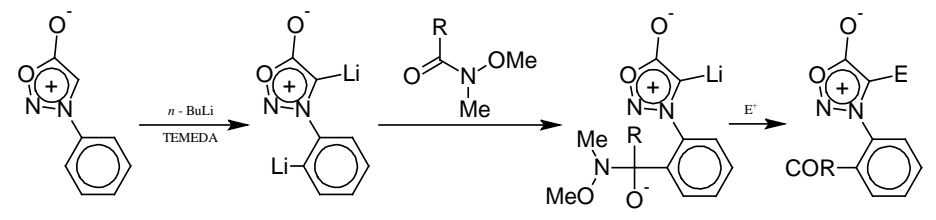

Scheme 6: One-pot o-acylation and subsequent sydnone 4-substitution

\section{The biological activity of sydnone}

The distinguished structure of sydnone having positive and negative charges along with its aromaticity and high lipophilicity enables it to react with biomolecules like DNA and enzymes. Consequently, sydnones exert a wide array of biological activities like antiinflammatory, analgesic, anti-arthritis, cytotoxicity, anti-parasite (malaria and leishmaniasis), antidiabetic, antioxidant, antimicrobial and nitric oxide donation [7]. In our present review, we will focus on the most studied and investigated biological activities.

\section{Anti-inflammatory activity}

The first report on the anti-inflammatory activity of sydnone-containing compounds was in 1974 by Wagner and Hill who reported that sydnones bearing 2-arylthioethyl or 2-arylsulfoxyethyl at the position N3 were promising scaffolds for designing new anti-inflammatory drugs. Structure-activity relationship revealed that a small lipophilic group like methyl or hydrogen atom at $\mathrm{C} 4$ was essential for the activity. Aromatic ring attached to the sulfur increased the potency with a maximum activity when both ortho positions were substituted with an electronegative atom. They found that the inhibition of arthritic swelling by 4-methyl-3-[2-(phenylthio) ethyl] sydnone was equal to hydrocortisone and phenylbutazone while 4-methyl-3-[2-(2,4dichlorophenylthio) ethyl] sydnone was six times stronger than hydrocortisone $[49,50]$.

Studies on the anti-inflammatory activity of more sydnone analogues were continued later by combining sydnone with other pharmacophores such as thiazole, pyrazoleand styryl ketone. It was found that 3-substituted-4-(thiazol-4-yl) sydnone had a weak to moderate activity [51]. On the other hand, the presence of 5arylpyrazole at $\mathrm{C} 4$ of the sydnone ring resulted in a favourable antiinflammatory activity as an anti-arthritis, anti-edema, and analgesic with less ulcerogenic side effects. For example, compound XXXV was found to be more effective than aspirin; $\mathrm{ED}_{50} 28.3$ vs. $81.4 \mathrm{mg} / \mathrm{Kg}$, respectively [52]. Sydnones containing substituted styrylketone were also investigated for their anti-inflammatory activity. Deshpande and his co-workers stated that some sydnonylstyrylketone XXXVI showed significant analgesic activity especially when there was an electron withdrawing group attached to the styryl moiety such as furyl, 4nitrophenyl, and 4-chlorophenyl. However, replacing the 4methoxyphenyl group in XXXVI by 3-chloro-4-flurophenyl enhanced the biological activity and decreased the ulcerogenicity compared to ibuprofen. Styryl-substituted sydnone exhibited a considerable analgesic activity in acetic acid-induced writhing but failed to show any significant activity in hot plate test suggesting that they act through peripheral rather than central effect $[53,54]$.

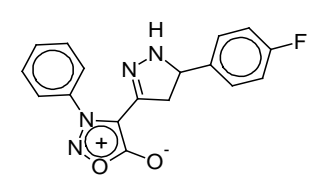

$X X X V$

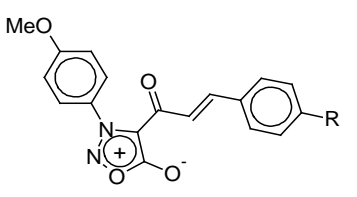

XXXVI

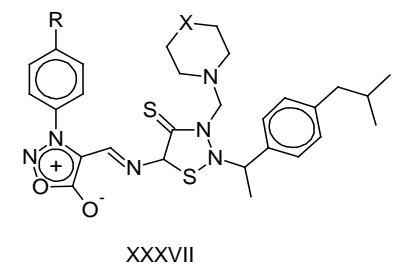

In other studies, sydnone ring hybrid with Mannich and Schiff bases XXXVII showed good anti-inflammatory and analgesic activities. Results showed that derivatives containing piperidine or morpholine had the highest anti-inflammatory activity in carrageenan-induced edemain rats with an analgesic activity comparable to the standard drug pentazocine [55]. A deeper insight into the mechanism of action of sydnones was provided by Kamble and his co-workers who postulated that benzophenone oxime compounds appended with sydnone directly inhibited phospholipase A2 (PLA2) by competing with the substrate at the binding cavity of the enzyme. Studies proved that PLA2 inhibitors can be as potent as steroidal anti-inflammatory drugs due to the reduction of lipid mediators which were usually secreted as a response to tissue injury [56]. Others found that 3-(4-chloro-3-nitro) sydnone mimicked the anti-inflammatory and immunosuppressive agents by reducing phagocytic activity, increasing superoxide anion production, inhibiting the production of nitric oxide, and declining interleukin-6 (IL-6) levels in peritoneal macrophages [57].

\section{Cytotoxic and anticancer activity}

In 1992, Grynberg and his co-researchers reported for the first time a successful usage of some sydnone derivatives as antitumor agents in vivo. They found that 3-(4-chloro-3-nitrophenyl) sydnone and 3-(4-pyrrolidono-3-nitrophenyl) sydnone exhibited a significant cytotoxic activity against sarcoma 180, Ehrlich carcinoma and B10MCII fibrous histiocytoma. Remarkably, only the first one showed a growth inhibitory activity against $L 1210$ leukaemia ascites tumors. They argued that the cytotoxic effect of sydnones might be due to inhibition of thymidine uptake by the cancerous cells [58].

Furthermore, sydnones were also linked to other pharmacologically active molecules to produce more potent cytotoxic agents. In this stream, sydnone-substituted chalcones were successfully synthesized and significantly inhibited the growth of Ehrlich ascites cells and Dalton's lymphoma ascites cells. Noteworthy, the existence of a methyl group on the chalcone moiety enhanced the survival of the experimental tumour-bearing animals while chloride atom produced a toxic compound [59]. A few years later, other 3-(halogen-substituted phenyl) sydnones were synthesized and tested against many cancer cell lines in vitro. It was found that a fluoride atom at the para position of the phenyl ring resulted in a sound antiproliferative activity against breast cancer MCF7, lung cancer NCI-H460 and central nervous system cancer $S F-268$. In contrast, replacing the halogen atom by other heterocyclic rings such as indole and isoindole was detrimental to the cytotoxic activity [60].

In addition, new stilbene-sydnone hybrids were prepared and found to decrease the viability and proliferation of cervical carcinoma (Hela), breast carcinoma (MCF7), colon carcinoma (SW620), pancreatic carcinoma (MiaPaCa2) and lung carcinoma (H460) cell lines in vitro. The most potent agents were found to have a chloride or methyl substituent on the stilbene moiety with a phenyl or methyl group at $\mathrm{C} 4$ of the sydnone ring like in structures XXXVIII and XXXIX [61]. Others reported a significant anticancer activity for new sydnones derivatized with imidazo $[2,1-b][1,3,4]$ thiadiazole and coumarin at $\mathrm{C} 4$ of the sydnone ring (XL) against HT-29 human colorectal adenocarcinoma cell line. They found that the hydrophobicity of $\mathrm{R}$ was crucial for the cytotoxic activity and the existence of a chlorine atom on the coumarin ring $\left(\mathrm{R}^{1}\right)$ sharply raised the activity to be comparable to that of cisplatin [62]. 
<smiles></smiles>

XXXVIII
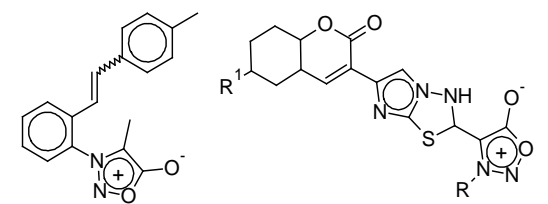

XXXIX

$X L$
Coordination complexes containing a metallic coordination center, sydnones, and other ligands were also synthesized and reported as new efficacious drugs such as the tridentate palladium Pd (II) complexes with thiosemicarbazone and phenyl sydnone (XLI). Sydnone and thiosemicarbazone bonded to the Pd (II) atom via the sydnone exocyclic oxygen, azomethine nitrogen and the sulfur atom afforded a yield of 40-60\%. The complexes exhibited a sound antiproliferative activity against human hepatocellular carcinoma (HepG2) and human cervical epithelial carcinoma (HeLa) cells. The $\mathrm{IC}_{50}$ of Pd-sydnone complexes were $0.77-2.25 \mu \mathrm{M}$ against HepG2 and 0.36-1.30 $\mu \mathrm{M}$ against HeLa whereas the $\mathrm{IC}_{50}$ of the standard drug 5fluorouracil were 6.94 and $0.71 \mu \mathrm{M}$, respectively [63].

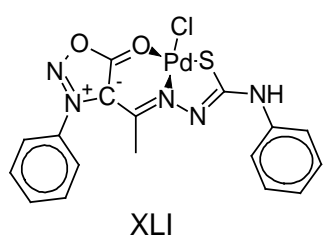

To further elucidate the mechanism of action of sydnone cytotoxicity, investigations were carried out on 3-(4-chloro-3nitrophenyl) sydnones (SYD-1). It was reported that mitochondria were the main targets of SYD-1 by lowering cellular energy production. Lipophilicity of SYD-1 enables it to interact with mitochondrial membrane resulting in altering of the components' redox state and finally shrinking of the mitochondria. It was also found that sydnone can lower the activity of glutamate dehydrogenase (GLDH). Being a nitric oxide donor, SYD-1 can interfere with the mitochondrial respiratory chain such as cytochrome oxidase leading to less oxygen utilization. Collectively, sydnone-induced cell death can be considered as a consequence of increased apoptosis [64]. Moreover, SYD-1 effectively reduced the oxidative stress of rat isolated mitochondria because it inhibited oxoglutarate-induced lipoperoxidation, reduced the formation/ opening of $\mathrm{Ca}^{+2}$-mediated permeability transition pores, and suppressed nicotinamide adenine dinucleotide phosphate-oxidase (NADPH) [65]. Recently, Galuppo et al. evaluated the biological activity of SYD-1 in tumour-bearing Wistar rats. They reported a significant decrease in tumor volume and tumor weight after $12 \mathrm{~d}$ of treatment by SYD-1 at a dose of $75 \mathrm{mg} / \mathrm{Kg}$. Histological examination of the tumor revealed coagulative necrosis and apoptotic spots. Therefore, they attributed sydnone activity to the activation of apoptosis pathways by decreasing the expression of anti-apoptotic enzyme Bcl-2 and increasing both apoptotic bodies and proapoptotic proteins (Bax and p53). However, splenomegaly was observed in the treated animals, and it was linked to sydnoneinduced extravascular hemolysis [66].

\section{Antimicrobial activity}

It has been demonstrated by numerous studies that sydnone derivatives have antibacterial and antifungal activities. Penicillin 3arylsydnone hybrids XLII were prepared by Naito and his colleagues from 3-arylsydnone-4-carboxylic acid and 6-aminopenicillanic acid. They were found to be active against penicillinase-producing bacteria strains. On the other hand, penicillin 3-alkylsydnones were inactive against the same resistant strains. It was postulated that the existence of a phenyl group at N3 of the sydnone ring resulted in sterical hindrance which protects the $\beta$-lactam carbonyl in a manner similar to that of oxacillin XLIII [67].

Sydnone-chalcone hybrids were also prepared and showed high antibacterial activity against gram-positive bacteria (Staphylococcus aureus) and weak activity against gram-negative bacteria (E. coli).

However, they did not exert antifungal activity. The existence of nitro group at the chalcone moiety enhanced the antibacterial activity. Remarkably, bromination of the $\alpha, \beta$-unsaturated ketone of the chalcone and the position $\mathrm{C} 4$ in the sydnone ring resulted in good bactericide molecules [68].

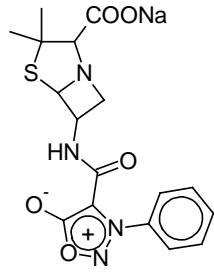

XLII

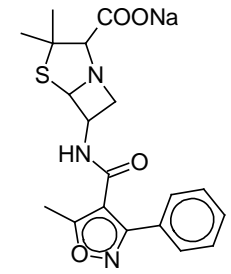

XLIII

Recently, more sydnone-containing antimicrobial agents were also prepared with more potent and wide spectrum activity. To mention some examples, 4-aminotriazine linked to the carbon $\mathrm{C} 4$ of the sydnone ring via mercaptoacetyl (XLIV), triazole (XLV)and benzothiazole (XLVI) linked to the sydnone ring via Mannich base showed promising antibacterial and antifungal activities even better than some known agents like nitrofurazone and ciprofloxacin [69-71].<smiles></smiles>

XLIV

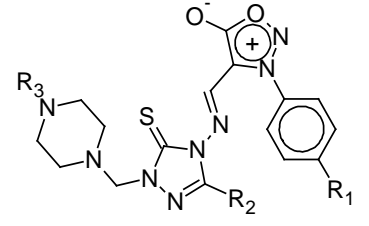

XLV<smiles></smiles>

$\mathrm{XLVI}$

Moreover, sydnone derivatives substituted at C4 with chlorosulfonyl or aminosulfonyl have attracted the attention of medicinal chemists as potential broad-spectrum antimicrobial agents by combining the biological properties of both pharmacophores in one entity. Asundaria, Patel and others synthesized many new sulfanoylcontaining sydnones (XLVII-LI) which showed weak to moderate antibacterial activities. Notwithstanding the nature of the substituents attached to the sulfanoyl group, none of the compounds exerted activity superior to the known antibiotic drugs [72-76].
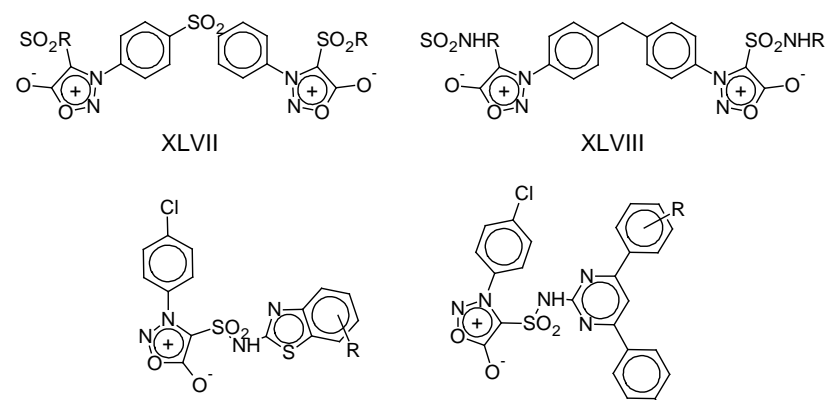

XLIX

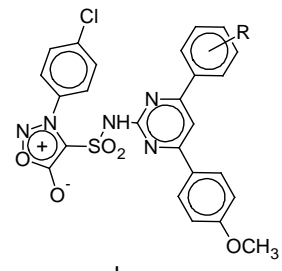

L

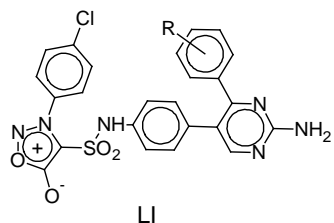

LI 


\section{Antioxidant activity}

Sydnone and its related compounds have been reported as antioxidant agents in various literature sources. In 1994, it was found that sydnone ring can enhance the antioxidant activity of chalcone by inhibiting lipid peroxidation and scavenging free radicals. Of interest, sydnone-substituted chalcones suppressed superoxide production by peritoneal macrophages in vivo in the presence of phorbol myristate acetate ester (PMA) which was linked to tumour generation [59]. Recently, 3-(halogen-substituted phenyl) sydnones combined with chalcone were reported as strong 2,2diphenyl-1-picrylhydrazyl (DPPH) free radical scavengers. The existence of fluorine and chlorine atoms at the phenyl ring of the sydnonyl moiety increased the antioxidant activity by nine folds compared to the commonly used antioxidant agent butyl hydroxy anisole (BHA) [77].

Additionally, sydnones substituted at C4 with thiazolidinone and thiazoline rings exhibited a moderate to potent DPPH free radical scavenging activity in vitro. Apparently, 2,3-dihydrothiazole ring linked to 3-phenylsydnone yielded powerful and rapid antioxidant compounds (LII) whose scavenging activities were comparable to that of $\alpha$-tocopherol. On the other hand, sydnones bearing 4-oxothiazolidine (LIII) were less active. The absence of $\mathrm{N}-\mathrm{H}$ group rendered the latter to be a weak scavenger [78, 79].

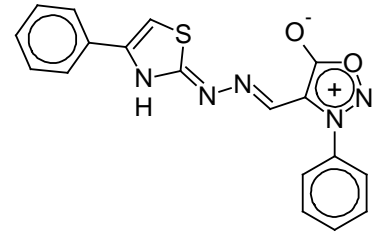

LII

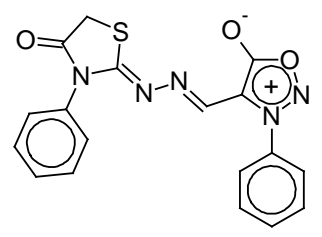

LIII

\section{Antimalarial activity}

In 1965, sydnone-based derivatives emerged as a new class of antimalarial agents. It was reported that 3-piperonylsydnone (LIV) and 3-phenylsydnone showed activity against Plasmodium berghei; the main parasite that causes malaria in mice. Nyber and Chen stated that 3-piperonylsydnone exhibited antimalarial activity when administered orally or subcutaneously at a dose of $10 \mathrm{mg} / \mathrm{Kg}$ with no toxic side effects even at a dose up to $500 \mathrm{mg} / \mathrm{Kg}$ [80]. Since 3phenylsydnone showed less activity and higher toxicity, it was of interest for other researchers to conduct a structure-activity analysis on the antimalarial activity of sydnone and piperonyl compounds. It was found that the $\mathrm{N}-\mathrm{N}$ bond is essential for the antiplasmodial activity either in the sydnone or in the piperonyl moiety as highlighted in structures LIV-LVII. However, 3piperonylsydnone was still the most active molecule among all tested compounds [81]. On the other hand, 4,4-bis (acetamidophenyl) sulfone derivatives were very potent antimalarial agents, while sydnone rendered it less active or inactive when they were combined in one structure LVIII [82]. Unfortunately, studies on sydnone-containing antimalarial agents were discontinued.

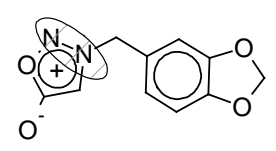

LIV

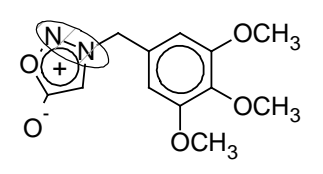

LV<smiles>CCCc1c(O)ccc(Cc2ccc3c(c2)OCO3)c1O</smiles>

LVI<smiles>NC(=S)NC1CCCC1c1ccc2c(c1)OCO2</smiles>

LVII

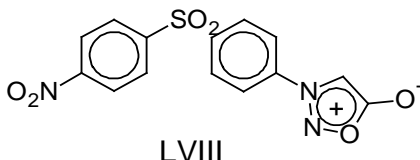

\section{CONCLUSION}

The wide spectrum of chemical and biological properties of sydnone and its derivatives reported in diverse literature sources makes it of paramount interest for chemists and pharmacologists. Thereupon, sydnones were considered chemically and medicinally versatile and robust molecules. They merit more exploration to furnish novel sydnone analogues linked to various substituents as potential scaffolds for the discovery of new drugs.

\section{CONFLICTS OF INTERESTS}

\section{Declared none}

\section{REFERENCES}

1. Baker W, Ollis WD. Mesoionic compounds. Quart Rev Chem Soc 1957;11:15-29.

2. Browne DL, Harrity JP. Recent developments in the chemistry of sydnones. Tetrahedron 2010;66:553-68.

3. Azarifar D, Bosra HG, Tajbaksh M. 1,3-Dibromo-5,5dimethylhydantoin (DBH) as an efficient promoter for acetylation of 3-arylsydnones in the presence of acetic anhydride under neutral conditions. J Heterocycl Chem 2007;44:467-9.

4. Ueng CH, Wang Y, Yeh MY. Structures of 4-acetyl-3-( $p$ tolyl)sydnone and 4-acetyl-3-phenylsydnone oxime. Acta Crystallogr Sect C: Struct Chem 1987;43:1122-5.

5. Earl JC, Mackney AW. The action of acetic anhydride on $\mathrm{N}$ nitrosophenylglycine and some of its derivatives. J Chem Soc (Resumed); 1935. p. 899-900.

6. Baker W, Ollis WD, Poole VD. Cyclic mesoionic compounds. Part III. Further properties of the sydnones and the mechanism of their formation. J Chem Soc (Resumed); 1950. p. 1542-51.

7. Chandrasekhar R, Nanjan MJ. Sydnones: a brief review. Mini Rev Med Chem 2012;12:1359-65.

8. Baker W, Ollis WD. Structure of the sydnones. Nature 1946;16:703.

9. Eade RA, Earl JC. The sydnones. a new class of compound containing two adjacent nitrogen atoms. J Chem Soc (Resumed); 1946. p. 591-93.

10. Kenner J, Mackay K. Structure of the sydnones. Nature 1946;21:909-10.

11. Applegate J, Turnbull K. The efficient synthesis of 3arylsydnones under neutral conditions. Synthesis 1988;1988:1011-2.

12. Daeniker HU, Druey J. Heilmittelchemische studien in der heterocyclischen reihe. 18. Mitteilung. über polymethylen-bissydnone und polymethylen-bis-hydrazine. Helvetica Chim Acta 1957;40:918-32.

13. Preston PN, Turnbull K. Approaches to the synthesis of compounds containing fused mesoionic rings. J Chem Soc, Perkin Trans 1977. p. 1229-33.

14. Stewart FHC. Cyclodehydration of $\alpha \alpha^{\prime}-d i-(N-2$-cyanoethyl- $N$ nitrosoamino)-glutaric Acid. J Chem Soc (Resumed) 1963. p. 2099-100.

15. Stewart FHC. Hydrolysis of $N$-alkyl- $N$-nitros-aspartic anhydrides. J Chem Soc (Resumed); 1963. p. 3158-60.

16. Azarifar D, Ghasemnejad-Bosra H, Ghorbani-Vaghei R, Tajbaksh M. $N, N, N^{\prime}, N^{\prime}$-tetrabromobenzene-1, 3-disolfonamide (TBBDS) as an efficient promoter for one-pot conversion of $\mathrm{N}$ arylglycines to sydnones in the presence of $\mathrm{NaNO}_{2} / \mathrm{Ac}_{2} \mathrm{O}$ under neutral conditions. Heterocycles 2006;68:2343-7.

17. Azarifar D, Ghasemnejad-Bosra H. Catalytic activity of 1,3dibromo-5,5-dimethylhydantoin $(\mathrm{DBH})$ in the one-pot transformation of $\mathrm{N}$-arylglycines to $\mathrm{N}$-arylsydnones in the presence of $\mathrm{NaNO}_{2} / \mathrm{Ac}_{2} \mathrm{O}$ under neutral conditions: subsequent bromination of these sydnones to their 4-bromo derivatives. Synthesis 2006;7:1123-6. 
18. Kier LB, Roche E. Molecular orbital calculations of the electronic structure of the sydnones. J Pharm Sci 1966;55:807-12.

19. Barnighausen $\mathrm{H}$, Jellinek F, Munnik Jt, Vos A. The structure of $N$ (p-bromophenyl) sydnone. Acta Cryst 1963;16:471-5.

20. Wang Y, Lee PL, Yeh MH. Structure of 3-(p-ethoxyphenyl) sydnone , $\mathrm{C}_{10} \mathrm{H}_{10} \mathrm{~N}_{2} \mathrm{O}_{3}$, and 3-(p-tolyl) sydnone, $\mathrm{C}_{9} \mathrm{H}_{8} \mathrm{~N}_{2} \mathrm{O}_{2}$. Acta Cryst Sect C: Cryst Str Comm 1984;40:1226-8.

21. Greco CV, O'Reilly BP. Mesoionic compounds vi metallation of sydnones. An estimation of acidity. J Heterocycl Chem 1970;7:1433-4.

22. Fan JM, Wang Y, Ueng CH. Electrostatic properties of sydnone derivatives. J Phys Chem 1993;97:8193-9.

23. Stewart FHC. The chemistry of the sydnones. Chem Rev 1964;64:129-47.

24. Kier LB, Roche EB. Medicinal chemistry of the mesoionic compounds. J Pharm Sci 1967;56:149-68

25. Patel YM, Patel KC. Synthesis and biological evaluation of new sydnone based derivatives. J Saudi Chem Soc 2012;8:113-8.

26. Lawson KD, Brey WS, Kier LB. Hydrogen chemical shifts of 3alkyl and 3-phenyl sydnones. J Am Chem Soc 1964;86:463-5.

27. Savaliya PP, Akbari VK, Patel KC. Studies on synthesis of some new sydnone containing compounds and their biological activities. Chem Sci Trans 2013;2:589-97.

28. Foster RS, Adams H, Jakobi H, Harrity JPA. Synthesis of 4Fluoromethylsydnones and their participation in alkyne cycloaddition reactions. J Org Chem 2013;78:4049-64.

29. Greco CV, Tobias J, Kier LB. Acylation of 3-phenylsydnone with carboxylic acids and phosphorus pentoxide. J Heterocycl Chem 1967;4:160-2.

30. Zhang ZB, Wu YR, Yin CL. The efficient synthesis of new sydnones containing fused ring. Synth Comm 2002;32:2203-7.

31. Tullis SA, Turnbull K. Acylation and aroylation of 3phenylsydnone. Synth Commun 1990;20:3137-44.

32. Turnbull K, George JC. Acylation of sydnones with acetic anhydride in the presence of montmorillonite K-10. Synth Commun 1996;26:2757-64.

33. Ghasemnejad-Bosra H, Haghdadi M, Gholampour-Azizi I. Nbromosuccinimide (NBS) as promoter for acylation of sydnones in the presence of acetic anhydride under neutral conditions. Heterocycles 2008;75:391-5.

34. Mahoney J, Turnbull K, Cubberley M. Bismuth triflate-catalyzed friedel-crafts acylations of sydnones. Synth Comm 2012;42:3220-9.

35. Balaguer AM, Selhorst R, Turnbull K. Metal triflate-catalyzed friedel-crafts acetylation of 3-phenylsydnone. Synth Commun 2013;43:1626-32.

36. Balaguer AM, Rumple AC, Turnbull K. Bismuth triflate catalyzed friedel-crafts acylation of 3-phenyl-and 3-(2carboalkoxyphenyl)sydnones under microwave irradiation. Org Prep Proced Int 2014;46:363-9.

37. Thoman CJ, Voaden DJ, Hunsberger IM. Direct formylation of sydnones. J Org Chem 1964;29:2044-5.

38. Yeh MY, Tien HJ, Huang LY, Chen MH. Sydnone compounds. Xx. The Synthesis and the schmidt reaction of 4-formyl-3arylsydnone. J Chinese Chem Soc 1983;30:29-37.

39. Greco CV, Mehta JR. Meso-Ionic compounds. XI, photochromic 4-halogeno-3-(3-pyridyl) sydnones. J Heterocycl Chem 1979;16:1059-60.

40. Dumitrașcu F, Drâghici C, Dumitrescu D, Tarko L, Râileanu D. Direct iodination of sydnones and their cycloadditions to form 5-iodopyrazoles. Liebigs Annalen 1997;1997:2613-6.

41. Dumitrașcu F, Mitan CI, Dumitrescu D, Drăghici C, Căproiu MT Steric effects on the sydnones reactivity. New sydnones and pyrazoles. Arkivoc 2002;2:80-6.

42. Nashashibi IF, Tumey JM, Owens BL, Turnbull K. Chlorination of 3-arylsydnones with iodine monochloride. Org Prep Proced Int 2017;49:59-63.

43. Fuchigami T, Ching-Sy C, Tsutomu N, Mou-Yung Y, Hsien-Ju T. Synthesis of sydnone compounds substituted by heteroatom groups at the 4-positions. Bull Chem Soc Japan 1986;59:483-6.

44. Fuchigami T, Ching-Sy C, Tsutomu N, Mou-Yung Y, Hsien-Ju T. Preparation of sydnone compounds substituted by thio and seleno functional groups at the 4-positions. Bull Chem Soc Japan 1986;59:487-91.
45. Turnbull K, Krein DM. The sydnone ring as an Ortho-director of lithiation. Dilithiation of 3-phenylsydnone and trapping by electrophiles. Tetrahedron Lett 1997;38:1165-8.

46. Turnbull K, Sun C, Krein DM. The sydnone ring as an Orthodirector of lithiation 2. Dilithiation of 3-phenylsydnone and regiospecific $O$-aryl acylation using $\mathrm{N}$-methoxy- $\mathrm{N}$ methylamides. Tetrahedron Lett 1998;39:1509-12.

47. Turnbull K, Krein DM. Formation of 4-Hydroxy-4-substituted Sydno[3,4-a] $(4 \mathrm{H})$ indoles via dilithiation of 3-(2Bromophenyl)sydnone. Synthesis 1996;10:1183- 4.

48. Turnbull K, Krein DM. Sydnone ring as an Ortho-director of lithiation, 3: one-pot regiospecific $O$-acylation and subsequent sydnone 4-substitution. Synth Commun 2009;39:2852-8.

49. Wagner H, Hill JB. Antiinflammatory sydnones 1. J Med Chem 1974;17:1337-8.

50. Hill JB, Ray RE, Wagner H, Aspinall RL. Antiinflammatory sydnones 2. J Med Chem 1975;18:50-3.

51. Upadhya KG, Badami BV, Puranik GS, Biradar VN, Nanjappa S. Synthesis and antiinflammatory activity of 3-substituted 4-(4'Thiazolyl)-sydnones. Arch Pharm 1980;313:684-8.

52. Satyabarayana K, Rao MNA. Synthesis of 4-[5-(Substituted aryl)-4,5-dihydro-1H-pyrazol-3-yl]-3-phenyl-sydnones as antiinflammatory, antiarthritic and analgesic agents. Eur J Med Chem 1995;30:641-5.

53. Deshpande SR, Pai KV, Pai RS. Design and synthesis of certain mesoionic sydnonyl styrylketones as potential nonsteroidal antiinflammatory agents. Arzneimittel-Forschung/Drug Res 2011;61:180-5.

54. Deshpande SR, Pai KV. Fluorine bearing sydnones with styryl ketone group: synthesis and their possible analgesic and antiinflammatory activities. J Enzyme Inhib Med Chem 2012;27:241-8

55. Nithinchandra, Kalluraya B, Aamir S, Shabaraya AR Regioselective reaction: synthesis, characterization and pharmacological activity of some new mannich and schiff bases containing sydnone. Eur J Med Chem 2012;54:597-604.

56. Kamble RR, Belgur SS, Aladkatti R, Khazi IA. Synthesis and evaluation of benzophenone oximes derivatized with sydnone as inhibitors of secretory phospholipase A2 with antiinflammatory activity. Chem Pharm Bull 2009;57:16-21.

57. Bizetto EL, Noleto GR, Echevarria A, Canuto AV, Cadena SM. Effect of sydnone SYD-1 on certain functions of LPS-stimulated macrophages. Mol Cel Biochem 2012;360:15-21.

58. Grynberg N, Gomes R, Shinzato T, Echevarria A, Miller J. Some new aryl-sydnones: effects on murine tumours. Anticancer Res 1992;12:1025-8.

59. Anto RJ, Kuttan G, Kuttan R, Sathyanarayana K, Rao MNA. Tumor-reducing and antioxidant activities of sydnonesubstituted chalcones. J Clin Biochem Nutr 1994;17:73-80.

60. Dunkley CS, Thoman CJ. Synthesis and biological evaluation of a novel phenyl substituted sydnone series as potential antitumor agents. Bioorg Med Chem Lett 2003;13:2899-901.

61. Butković K, Marinić Ž, Šindler-Kulyka M. Synthesis of 3-(oStilbenyl)-sydnone and 3-(o-Stilbenyl)-4-substituted-sydnone derivatives and their antitumor evaluation. Arkivoc 2011;10:115.

62. Tegginamath G, Kamble RR, Taj T, Kattimani PP, Meti GY. Synthesis of novel imidazo[2,1-B][1,3,4]thiadiazoles appended to sydnone as anticancer agents. Med Chem Res 2013;22:4367-75.

63. Shih MH, Chen JC, Lin GL, Lin TT, Sun MH. Novel synthesis of palladium (II) complexes derived from 3-arylsydnone-4carbaldehyde $\mathrm{N}$-phenylthiosemicarbazones and biological activity. J Pharm Pharmacol 2014;66:73-83.

64. Halila GC, de Oliveira MB, Echevarria A, Belém AC, Rocha ME, Carnieri EG, et al. Effect of sydnone SYD-1, a mesoionic compound, on energy-linked functions of rat liver mitochondria. Chem Biol Interact 2007;169:160-70.

65. Gozzi GJ, Pires AD, Martinez GR, Rocha ME, Noleto GR, Echevarria $A$, et al. The antioxidant effect of the mesoionic compound SYD-1 in mitochondria. Chem Biol Interact 2013;205:181-7.

66. Galuppo LF, dos Reis Lívero FA, Martins GG, Cardoso CC Beltrame OC, Klassen LMB, et al. Sydnone 1: a mesoionic compound with antitumoral and haematological effects in vivo. Basic Clin Pharmacol Toxicol 2016;119:41-50. 
67. Naito T, Nakagawa $S$, Takahashi $K$, Masuko $K$, Fujisawa $K$ Synthesis of 6-aminopenicillanic acid derivatives 3. 6-(3Substituted-sydnone-4-carboxamido)-penicillanates. J Antibiot 1968;21:290.

68. Moustafa MAA, Eisa HM. Synthesis and antimicrobial activity of 3-(Substituted-phenyl)-sydnones. Arch Pharm 1992;325:397401.

69. Hegde JC, Girisha KS, Adhikari A, Kalluraya B. Synthesis and antimicrobial activities of a new series of 4-S-[4-amino-5-oxo6-substituted benzyl-4,5-dihydro-1,2,4-triazin-3yl]mercaptoacetyl-3-arylsydnones. Eur J Med Chem 2008;43:2831-4.

70. Savaliya PP, Akbari VK, Modi JA, Patel KC. Synthesis, characterization, and antimicrobial screening of some mannich base sydnone derivatives. Med Chem Res 2013;22:5789-97.

71. Rahiman MA, Kalluraya B. Synthesis, characterization, antimicrobial and anthelmintic activity of some sydnone- $\mathrm{N}$ mannich bases. J Indian Counc Chem 2008;25:10-4

72. Asundaria ST, Patel KC. Synthesis and characterization of some sulfonamide based bis-sydnones and their in vitro antimicrobial activity. Indian J Chem Sect B: Org Chem Incl Med Chem 2010;49:960-4.

73. Asundaria ST, Patel KC. Synthesis, Characterization, and Antimicrobial Studies of Bissydnones Based on 4,4'Diaminodiphenyl Methane. Synth Comm 2010;40:1899-906.

74. Asundaria ST, Patel KC. Synthesis, characterization and antimicrobial activity of thiazole, benzothiazole and pyrimidine derivatives bearing sydnone moieties. Pharm Chem J 2012;45:725-31.

75. Asundaria ST, Pannecouque C, De Clercq E, Patel KC. Sydnone sulfonamide derivatives as antibacterial, antifungal, antiproliferative and anti-HIV agents. Pharm Chem J 2014;48:260-8.

76. Patel YM, Patel KC. Synthesis and biological evaluation of new sydnone based derivatives. J Saudi Chem Soc 2015;19:193-9.

77. Bhosale SK, Deshpande SR, Wagh RD. Ultrasound assisted one pot synthesis, spectral, antimicrobial and antioxidant studies of novel 4-[1-oxo-3-(substituted phenyl)-2-propenyl]-3substituted phenyl sydnones. Asian J Chem 2015;27:3063-8.

78. Shih MH, Ke FY. Syntheses and evaluation of antioxidant activity of sydnonyl substituted thiazolidinone and thiazoline derivatives. Bioorg Med Chem 2004;12:4633-43.

79. Shih MH, Su YS, Wu CL. Syntheses of aromatic substituted hydrazino-thiazole derivatives to clarify structural characterization and antioxidant activity between 3arylsydnonyl and aryl substituted hydrazino-thiazoles. Chem Pharm Bull 2007;55:1126-35.

80. Nyberg WH, Cheng CC. 3-Piperonylsydnone. A new type of antimalarial agent. J Med Chem 1965;8:531-3.

81. Boots SG, Cheng CC. Structural modification studies of 3piperonylsydnone i. synthesis of piperonyl-substituted pyrazoles, isoxazoles, triazoles, oxadiazoles and thiadiazoles. J Heterocycl Chem 1967;4:272-83.

82. Popoff IC, Singhal GH, Engle AR. Antimalarial agents 7 . Compounds related to 4,4'-Bis (aminophenyl) sulfone. J Med Chem 1971;14:550-1.

\section{How to cite this article}

- Abdualrahman Mohammed Abdualkader, Muhammad Taher Nik Idris Nik Yusoff. Mesoionic sydnone: a review in their chemical and biological properties. Int J Pharm Pharm Sci 2017;9(8):1-9. 